\title{
Microfluidic High-Throughput Imaging and Automated Lineage Tracking of Yeast
}

D. Falconnet*, M. Ricicova*, A. Quiring*, A. Niemistö**, R.J. Taylor***, T. Galitski**, I. Shmulevich**, C.L. Hansen*,**

*Department of Physics and Astronomy and the Center for High-Throughput Biology, University of British Columbia, Vancouver, BC V6T 1Z4

**Institute for Systems Biology, Seattle, WA 98103

Heterogeneity in cellular response is present across all organisms, from E. coli to stem cells, and is a critical challenge to deciphering biological responses. Even cells with identical genotype and cell history respond differently to stimuli. This variability is obscured in many conventional biochemical assays which necessarily average the response of large ensembles of individual cells. A particularly interesting source of cellular variation is epigenetic differences or heritable cell states, possibly arising from correlated protein and mRNA levels between cells and their progeny. Quantitative analysis of large numbers of single cells is critical to investigating these effects and is well-suited to quantitative time-lapse microscopy. Technical challenges in conventional systems include the need to track single cells through time, difficulty in reconstructing lineage relationships, and crude temporal control over the chemical microenvironment. Here we describe a microfluidicbased imaging platform and related image analysis pipeline optimized for such high-throughput experiments with yeast. Using this system we investigate heritable sources of noise in the mitogenactivated protein kinase (MAPK) cascade which governs pheromone response in Saccharomyces Cerevisiae.

Microfluidic devices can be used to improve long-term cell imaging through confinement, to precisely control chemical conditions in space and time, and to execute multiple parallel experiments in a compact high-throughput format [1][2]. Our microfluidic system (Fig. 1) is constructed from multiple layers of PDMS and contains 128 chambers, each capable of holding up to 1000 cells, arranged in eight columns and sixteen rows [3]. Each column contains a single genotype and each row a single chemical condition. The cells are immobilized in agarose gel (Fig. 2), allowing the acquisition of high-resolution fluorescent images in three colors and the tracking of cells over up to 16 hours with time resolution of 20 minutes. The flow of cells and exchange of media is automated using integrated micromechanical valves, enabling the programmable and dynamic modulation of the microenvironment. Yeast strains are transformed with fluorescent proteins to allow for tracking of transcriptional response and noise while further facilitating the robust association of daughters with mothers. Custom MATLAB image analysis software is used to process over 60,000 images per experiment in order to segment cells, track cells through time, construct lineages, and correlate this to activity of the pheromone response pathway.

Preliminary investigations with this system reveal that a switch-like morphological response in yeast pheromone signaling is correlated with the gene expression and that these responses are influenced by lineage-dependent effects. Our system's precise chemical control, high throughput, and ability to 
immobilize cells may be used in the future to explore similar properties of other pathways and cell types.

\section{References}

[1] G. Charvin et al., PLoS ONE. 3(1): e1468 (2008) doi:10.1371/journal.pone.0001468.

[2] A.C. Rowat et al., PNAS. 106 (2009) 18149.

[3] R.J. Taylor et al., PNAS. 106 (2009) 3758.

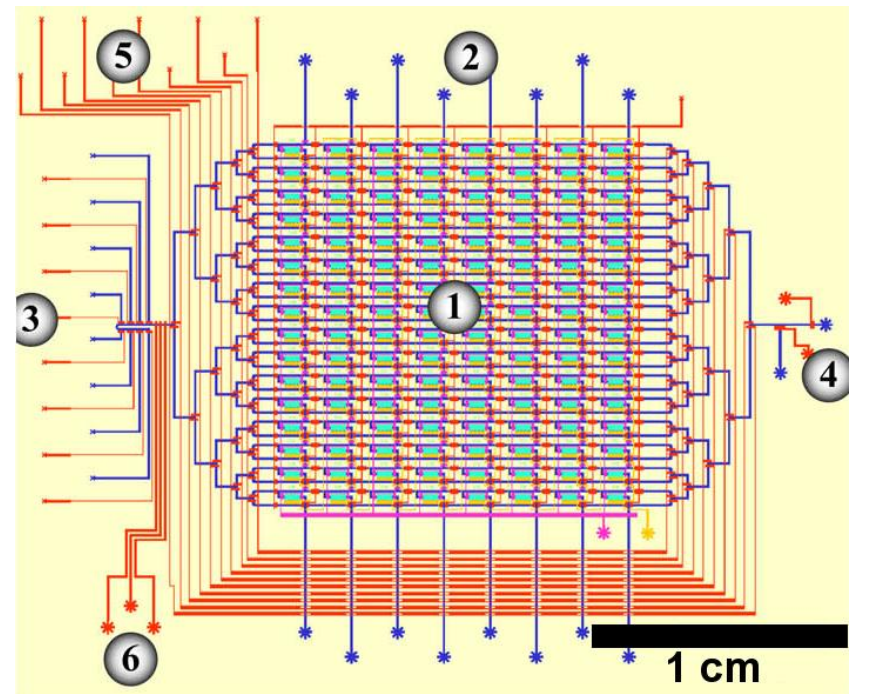

FIG. 1. Microfluidic chip design. (1) Array of imaging chambers (8 columns and 16 rows), (2) column inlets for loading different strains, (3) chemical inlets, (4) waste outlets, (5) fluidic multiplexer to address the chemicals to certain rows, (6) peristaltic pump for mixing input chemicals.

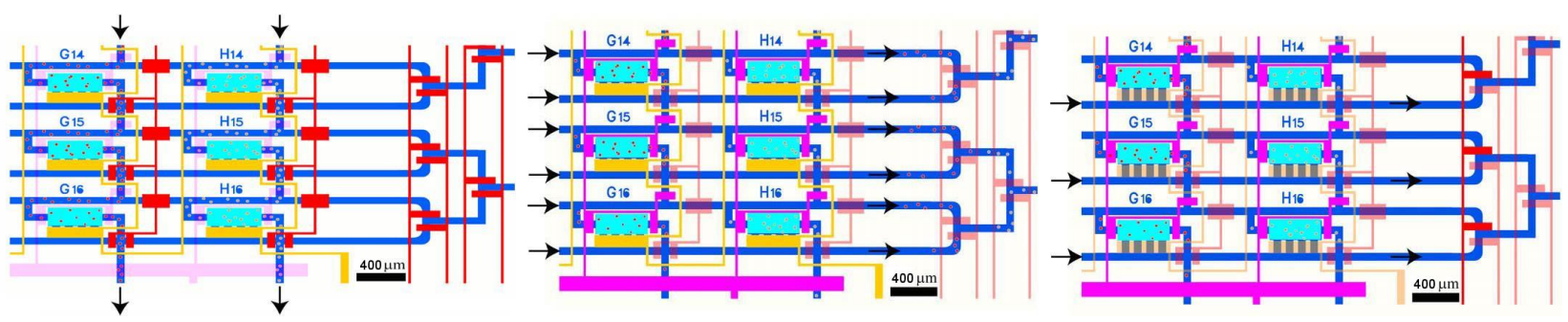

FIG. 2. Cell loading and immobilization. (Left) Columns are isolated from each other using column separation valves (red) and cells in suspension with liquid agarose are loaded into each column. Diffusion valves (yellow) are closed while the chamber valves (pink) are open. (Middle) Actuation of chamber valves (pink) isolates each chamber and row. The chip is cooled and the agarose sets, immobilizing the cells. (Right) The diffusion valves are opened to allow media exchange between chambers and row channels. 